\title{
Designing Good Multi-Dimensional Constellations
}

\author{
Marko Beko and Rui Dinis
}

\begin{abstract}
In this letter we consider the design of multidimensional compact constellations that minimize the average symbol energy for a given minimum Euclidian distance between constellation points. We formulate the constellation design as a non-convex quadratically constrained quadratic programming. We propose a simple and efficient optimization method, which offers good solutions for small to medium sized constellations.
\end{abstract}

Index Terms $-M$-ary constellations, optimization.

\section{INTRODUCTION}

I $\mathrm{N}$ a digital communication system a set of bits is used to select a symbol to be transmitted. This symbol belongs to a given constellation with $M$ symbols. To allow good power efficiency we want to minimize the transmit power for a given error rate. Although the performance depends on the combined effects of channel coding and constellation, the overall gain can be decomposed in coding gain and shaping gain, the later associated to the constellation design addressed in this work, which can be optimized separately with minor losses [1]). This means that the distance between symbols within the constellation should be as high as possible, for a given average bit energy. The constellation symbols can be regarded as vectors in a $N$-dimensional space and the design of good constellations can be regarded as an optimization problem.

In general, the larger the number of dimensions the worse the spectral efficiency, although we can improve the power efficiency. On the other hand, increasing the constellation size while maintaining the number of dimensions leads to higher spectral efficiency. If we do not have bandwidth constraints it is known that we can achieve excellent power efficiency by using orthogonal constellations. Since orthogonal constellations have non-zero mean, we can improve the power efficiency of an orthogonal constellation by employing simplex constellations, where the orthogonal constellation is shifted to have a zero mean, or bi-orthogonal constellations, where we have a vector and its symmetric in each dimension [2].

Manuscript received March 19, 2012. The associate editor coordinating the review of this letter and approving it for publication was G. Colavolpe.

M. Beko is with the Universidade Lusófona de Humanidades e Tecnologias and UNINOVA (e-mail: mbeko@uninova.pt).

Rui Dinis is with the Instituto de Telecomunicações and DEE, FCT-UNL.

This work was partially supported by Fundação para a Ciência e a Tecnologia under Projects PTDC/EEA-TEL/099973/2008 ADCOD, PEst-OE/EEI/UI0066/2011, PEst-OE/EEI/LA0008/2011 and PTDC/EEATEL/115981/2009OPPORTUNISTIC-CR, and Ciência 2008 Post-Doctoral Research grant PEst-OE/EEI/LA0008/2011.

M. Beko is also a collaborative member of the Instituto de Sistemas e Robótica - Instituto Superior Técnico, Av. Rovisco Pais, 1049-001 Lisboa, Portugal, and SITI/ILIND/COFAC: R\&D Unit of Informatics Systems and Technologies, Instituto Lusófono de Investigação e Desenvolvimento (ILIND), COFAC, Lisboa, Portugal.

Digital Object Identifier 10.1109/WCL.2012.032312.120203
On the opposite side we have the highly bandlimited case where we want high spectral efficiency and, consequently, constellations with a large number of points per dimension. Typically we employ constellations with one or two dimensions. It is known that regular PAM constellations with zero average are the best linear (i.e, with one dimension) constellations. For 2-D constellations we usually employ QAM or APSK constellations. Although QAM constellations can have better shaping gains than APSK constellations, the mutual information of the later ones might be slightly higher [3], [4]. Even in terms of shaping gains, QAM constellations are the best 2-D constellations since we could reduce the average symbol energy by using approximately circular instead of a square shape. The best known 2-D constellations are Voronoi constellations [5], where we place the points over a hexagonal grid [6]. The gain on the average symbol energy when we move from a $N$-dimension cube to a $N$ dimension sphere, usually denoted shaping gain, is about $1.53 \mathrm{~dB}$ for large multi-dimensional constellations [6]. By employing generalized QAM constellations we can achieve good shaping gains [7] and even better gains with Voronoi constellations [8]. In [9], Foschini et al constructed good codes in 2-D using a gradient descent (GD) algorithm. The problem of constructing compact constellations in 3-D and 4-D was addressed in the past by J. A. Sloane et al using a number of different techniques, which include quadratic programming among others [10], [11]. Most constellations are designed to have the points over a grid so as to give them some structure that simplifies its implementation [12]. However, this sets some restrictions in the constellation design that might limit the achievable gains. If the constellation does not have a clear structure the mapping and demapping operations can be done through table-lookup procedures, something that is not difficult with present day technology, even for high bit rates. Therefore, it is important to know the achievable power efficiency of multi-dimension constellations and how to design good multi-dimension constellations. Unfortunately, obtaining the best constellations with more than two dimensions is a difficult problem.

In this letter we consider the design of size- $M$ constellations with $N>2$ dimensions. Instead of the extreme cases where the constellation size is similar or not much higher than the number of dimensions (i.e., for the case without bandwidth limitations) or the case where the constellation size is much higher than the number of dimensions (i.e., the case with very high spectral efficiencies), we consider intermediate cases. We formulate the constellation design as a non-convex optimization problem which is tackled by solving a sequence of convex optimization problems where we minimize the convex quadratic objective function subject to a set of linear inequality 